\title{
Cardiac Resynchronization with His-CRT in a patient with severe heart failure and Scimitar syndrome.
}

\author{
Quinton Yang ${ }^{1}$, Deep Raja ${ }^{1}$, Simon Stolcman ${ }^{1}$, S P Abhilash ${ }^{1}$, Robert Greenough ${ }^{1}$, Walter \\ Abhayaratna ${ }^{1}$, Ren Tan $^{1}$, and Rajeev Pathak ${ }^{1}$ \\ ${ }^{1}$ Canberra Hospital
}

May 8, 2020

\begin{abstract}
Cardiac resynchronization therapy with His-bundle pacing (His-CRT) is evolving rapidly as a viable cardiac device strategy for the treatment of severe chronic heart failure. The success of this technique in patients with congenital heart disease is facilitated by advanced integrated imaging modalities. We report a case of His-CRT pacing for the management of a patient with heart failure with severely reduced ejection fraction, left bundle branch block and congenital heart disease characterized by Scimitar syndrome with cardiac dextroposition. We highlight the contribution of integrated imaging modalities to guide accurate lead positioning.
\end{abstract}

\section{Introduction:}

Scimitar syndrome is a rare pulmonary venolobar syndrome that is characterized by anomalous pulmonary vein $(\mathrm{PV})$ drainage of the right lung, most frequently into the inferior vena cava. Associated congenital abnormalities include right lung hypoplasia and cardiac dextroposition, with or without structural heart defects. We describe a case of a patient with Scimitar syndrome and severe chronic heart failure with left bundle branch block (LBBB) who underwent cardiac resynchronization therapy with His-bundle pacing (HisCRT), resulting in significant improvements in functional capacity and left ventricular (LV) function. To the best of our knowledge, this is the first reported case of His-CRT in Scimitar syndrome. We highlight the contribution of integrated imaging modalities to guide accurate lead positioning.

Case Report:

A 71-year-old woman presented with decompensated biventricular heart failure in 2015, thought to be due to acute myocarditis. The CT chest revealed a partial anomalous right upper pulmonary venous drainage, right hypoplastic lung and cardiac dextroposition, findings consistent with Scimitar syndrome. Her ECG revealed LBBB with a QRS duration of 196ms (Figure 1A). She was referred to our institution due to persistent NYHA III-IV symptoms with a severely reduced LV ejection fraction (LVEF) of $25 \%$. Her vasoactive medical therapy was limited by hypotension. Cardiac resynchronization therapy (CRT) was indicated.

In planning for this complex procedure, cardiac $\mathrm{CT}$ angiogram and pulmonary venogram revealed right lung hypoplasia and mediastinal shift to the right thoracic cavity leading to dextroposition of the heart chambers. Her left ventricle was severely dilated. The right upper pulmonary vein drained into the inferior vena cava (Figure 1C). The coronary sinus had a high superior take-off but did not demonstrate a left sided superior vena cava. Her previous right heart catheterisation revealed moderately elevated pulmonary artery pressures but only minor left to right shunting with a Qp:Qs of 1.24 . The peak systolic and mean pulmonary artery (PA) pressures were $62 \mathrm{mmHg}$ and $32 \mathrm{mmHg}$, respectively. 
The cardiac CT scan was used to register with the CARTOSEG modality of the CARTO (CARTOß 3 Version 6) three-dimensional electroanatomical mapping system. The ventricular septum was found to be rotated anti-clockwise by $60^{\circ}$ due to dextroposition. Fluoroscopic view of RAO 20-30 helped us in orienting the interventricular septum perpendicularly (Figure $2 \mathrm{~A}$ ).

The patient was electively intubated. Using NAVISTAR ${ }^{\circledR}$ DS Bi-Directional Catheter (4mm 7French Curve D-F) and CARTO 3D Mapping system ${ }^{\mathrm{TM}}$ (Version 6), images were created, aligned, and verified with the cardiac CT geometries on the CARTOSEG. Important landmarks like the right atrial appendage, tricuspid annulus, the ostium of the coronary sinus and the His-bundle were tagged (Figure 2B). All the obtained geometries were integrated onto the fluoroscopic screen using the CARTOUNIVU modality (Figure 3A-D). A quadripolar catheter was placed in the RV for back-up pacing.

Left-sided delto-pectoral pocket was created. Three separate venography-guided extra-thoracic axillary venous accesses were secured. The axillary vein was then cannulated and a Medtronic(r) $62 \mathrm{~cm} 6935 \mathrm{M}$ high voltage lead advanced to the RV apical septum. CARTOUNIVU was used to advance the RV lead to the desired location of the lower septum and the position was confirmed in the RAO $30^{\circ}$ and antero-posterior (AP) views (Figure 3A). The cannulation of the coronary sinus (CS) was then attempted. The ostium of the CS was angulated at $90^{\circ}$ from usual anatomy, with a sharp superior and anterior tortuosity before reaching the body of the CS (Figure 2B). Neither the standard nor Amplatz curve Attain Command and the $90^{\circ}$ and $135^{\circ}$ Attain Select II catheters were successful in cannulating the CS. However, we were successful in advancing a steerable decapolar diagnostic electrophysiology catheter into the CS on which the long sheath was telescoped. We identified 3 tributaries targeted for lead advancement. Unfortunately, all the tributaries had angulation and tortuous take-off precluding successful advancement of any hardware. After multiple attempts with various tools, LV lead placement was abandoned, and His-bundle pacing was attempted.

Placement of the His-bundle lead was guided by the tagged His-bundle anatomy, which was integrated onto the fluoroscopic screen with the CARTOUNIV. Fluoroscopic angles of RAO $30^{\circ}$ and AP were helpful in guiding the Medtronic(r) C315 catheter to the perceived His-bundle location (Figure 2C). Mapping using the Medtronic(r) $69 \mathrm{~cm} 3830$ pacing lead recorded His-bundle potential at the targeted region (Figure 2D). Passive pacing at the distal His region resulted in a threshold of $5 \mathrm{~V}$ at $1 \mathrm{~ms}$ with correction of LBBB (Figure $2 \mathrm{E})$. This resulted in non-selective capture of the His-bundle with complete correction of the LBBB. The threshold for LBBB correction was $1.5 \mathrm{~V}$ at $1 \mathrm{~ms}$ and the capture threshold was $0.75 \mathrm{~V}$ at $0.4 \mathrm{~ms}$. Finally, the atrial lead was then placed at the right atrial appendage guided again by CARTOUNIVU. A Medtronic(r) Amplia MRI Quad CRT-D device was secured in the pre-formed left pectoral pocket. The fluoroscopic time was 117 minutes, and procedural time was 350 minutes. The His-CRT was programmed with a LV-RV delay of $-80 \mathrm{~ms}$ and a programmed output of $3.5 \mathrm{~V}$ at $1.0 \mathrm{~ms}$ for the His bundle lead. The device mode was set to DDDR 60bpm. The following changes in the 12-lead ECG parameters suggested successful correction of the LBBB by capturing the His-bundle: 1. Narrowing of QRS of $54 \mathrm{~ms}$ (from $196 \mathrm{~ms}$ to $142 \mathrm{~ms}$ ); 2. Change in mean QRS axis (from $+16^{\circ}$ to $+42^{\circ}$ ), and; 3. Decrease in LV activation time (measured in V5 from 90ms to $60 \mathrm{~ms}$ ) (Figure 1B).

The patient returned 6 months post His-CRT-D implantation for review. The lead parameters were satisfactory. She was $99.7 \%$ paced. The LBBB correction threshold of the His lead was $2.00 \mathrm{~V}$ at $1 \mathrm{~ms}$. The patient had an improvement in her functional status from NYHA Class III-IV to Class II, associated with a $36 \%$ relative increase in LVEF (from $25 \%$ to $34 \%$ ).

\section{Discussion:}

In this case report, we have demonstrated successful cardiac resynchronisation using His-bundle pacing in a patient with Scimitar syndrome with subsequent improvement in the patient's heart failure symptoms. Utilization of integrated image modalities using CARTOSEG and CARTOUNIVU software helped us to achieve successful cardiac resynchronization despite the difficult anatomy with respect to the cardiac chambers as well as the CS tributaries. To the best of our knowledge, our case is the first description of His-CRT implantation in Scimitar syndrome with image integration tools. 
Image integration using three-dimensional electro-anatomical mapping systems was crucial for understanding the Scimitar syndrome complex cardiac anatomy given the right lung hypoplasia and right sided dextroposition of the heart ${ }^{1}$, and in advancing the lead to the key cardiac landmarks. In our patient, L-transposition of great arteries (L-TGA) and left sided valvular abnormalities were specifically ruled out.

The use of CARTOSEG and CARTOUNIVU image integration modules has previously been shown to reduce fluoroscopy time and procedural complications. ${ }^{2}$ In our case, planning with CARTOSEG allowed orientation to the inter-ventricular septum in unconventional fluoroscopic views. Predefining and integrating the location of the His-bundle potentially reduced the procedural time.

Cardiac resynchronization with His-bundle pacing (His-CRT) is evolving rapidly as a viable strategy and as an alternative to the LV lead implanted through the CS (conventional CRT). ${ }^{3,4}$ This has added a new dimension to patients where placement of an LV lead may be technically challenging as in our case. It is possible to correct the focal and proximal LBBB at the level of the His conduction system by pacing distal to the block as shown in our case.

In congenital heart diseases, the perceived difficulties of His-bundle pacing are related either to the cardiac malrotation as observed in our case or due to varied positions of the AV node conduction axis. Nevertheless, His-bundle pacing for complete heart block has been achieved in congenital anomalous hearts like in persistent left superior vena cava (LSVC) ${ }^{5}$, dextrocardia ${ }^{6}$, Ebstein's anomaly ${ }^{7}$ and in L-TGA ${ }^{8}$. In a recent case series of patients with intraventricular conduction defects and L-TGA, His-bundle pacing was preferred over conventional CRT given the CS may be impossible to reach without compromise to procedural success rates and subsequent functional improvements. ${ }^{9}$ As the technology evolves, we will expect successful His-bundle pacing in all ranges of congenital heart diseases. The extent and duration of the desired reverse remodelling of the LV remains to be seen in our case.

\section{Conclusion:}

Cardiac resynchronization therapy with His-bundle pacing is feasible in patients with Scimitar syndrome. The use of advanced three-dimensional cardiac mapping integrated with fluoroscopy using software such as CARTOSEG and CARTOUNIVU assists in locating the critical cardiac landmarks when challenged with unconventional fluoroscopic views. Our case adds to the growing literature of successful His-bundle pacing for CRT in patients with congenital heart diseases.

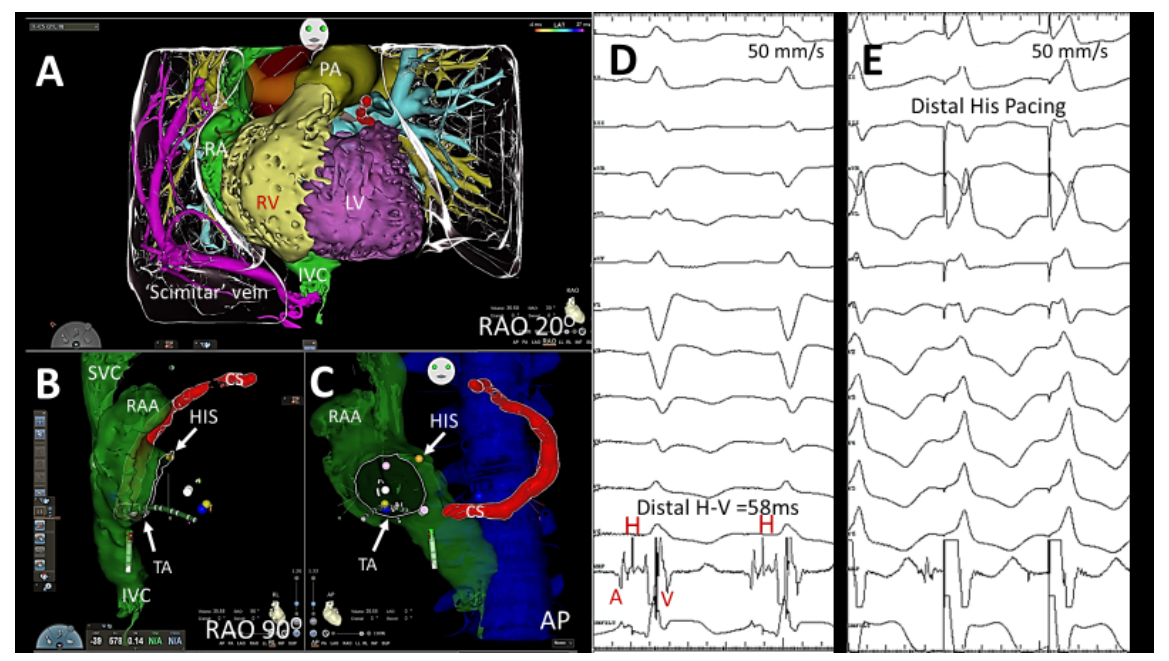




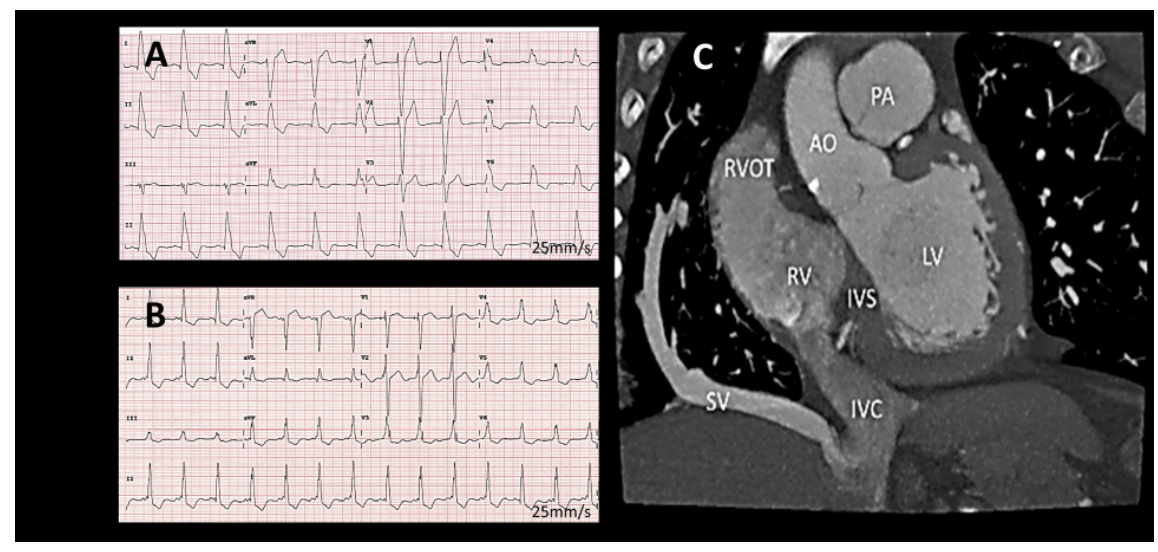

Figure 1: Panel A shows the 12-lead ECG intrinsic LBBB with QRS duration of 196ms (the precordial leads were placed on the right side due to dextroposition of the heart chambers); Panel B shows the 12-lead ECG after His-CRT with QRS duration of $146 \mathrm{~ms}$ and correction of the LBBB; Panel C shows the cardiac CT scan demonstrating the 'Scimitar' vein - anomalous drainage of the right pulmonary vein into the IVC. $\mathrm{RA}=$ right atrium, $\mathrm{RV}=$ right ventricle, $\mathrm{LV}=$ left ventricle, $\mathrm{PA}=$ pulmonary artery, $\mathrm{IVC}=$ inferior vena cava, $\mathrm{SV}=$ Scimitar vein.

Figure 2: Panel A shows the CARTOSEG modality wherein the Cardiac CT was integrated onto the CARTO electro-anatomical mapping system. Note the perpendicular orientation of the interventricular septum at RAO 20; Panels B and C show the geometries created by an electrophysiology catheter which was integrated with the cardiac CT scan. His bundle (yellow marker) was tagged (white arrow). Panel D and $\mathrm{E}$ show the intracardiac electrograms observed with the lead. The distal $\mathrm{H}-\mathrm{V}$ was $58 \mathrm{~ms}$. Pacing at this site resulted in correction of the $\mathrm{LBBB} ; \mathrm{AP}=$ antero-posterior, $\mathrm{RAO}=$ right anterior oblique, $\mathrm{LAO}$ $=$ left anterior oblique, $\mathrm{TA}=$ tricuspid annulus, $\mathrm{CS}=$ coronary sinus, $\mathrm{RA}=$ right atrium, $\mathrm{RAA}=$ right atrial appendage, $\mathrm{RV}=$ right ventricle, $\mathrm{RVOT}=$ right ventricular outflow tract, $\mathrm{LV}=$ left ventricle, $\mathrm{PA}=$ pulmonary artery, IVC $=$ inferior vena cava.

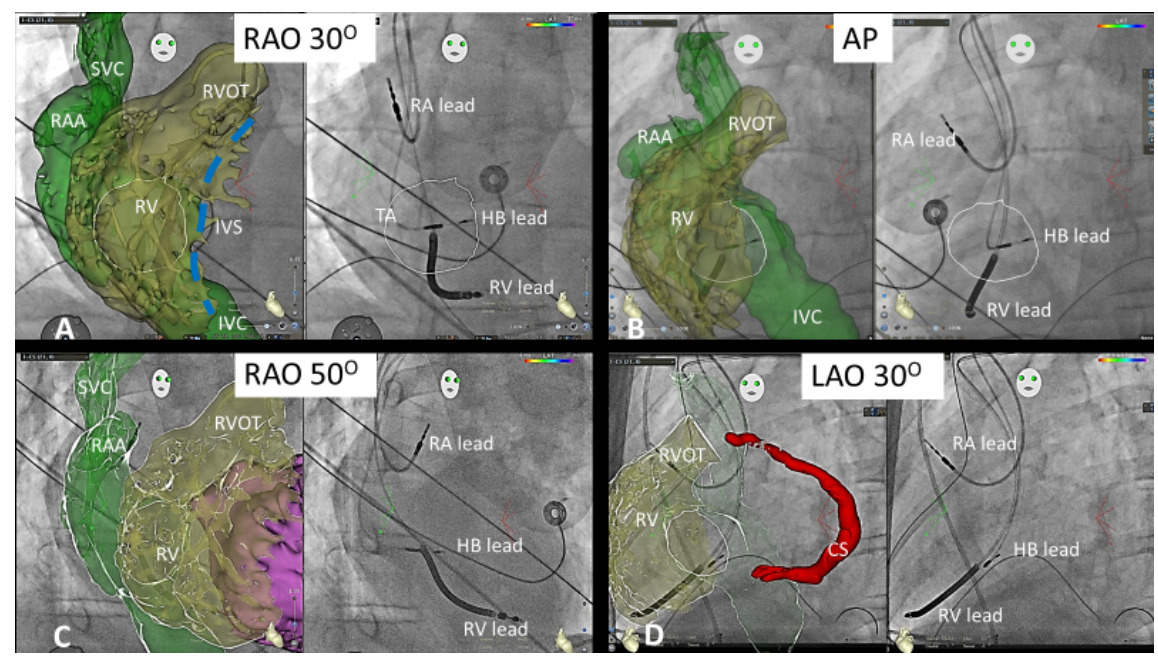

Figure 3: Demonstrating the CARTUNIVU modality wherein the image created using the CARTO was integrated onto the fluoroscopic screen. Each panel shows the location of the heart chambers on the left screen and final lead positions on the right. Panels A-D show RAO $30^{\circ}$, AP, RAO $50^{\circ}$ and LAO $30^{\circ}$ views, respectively. Note the perpendicular orientation of the His bundle lead and RV lead implant towards the 
interventricular septum, and atrial lead in right atrial appendage. $\mathrm{AP}=$ antero-posterior, $\mathrm{RAO}=$ right anterior oblique, $\mathrm{LAO}=$ left anterior oblique, $\mathrm{TA}=$ tricuspid annulus (white outline), $\mathrm{CS}=$ coronary sinus, $\mathrm{HB}=$ His bundle $\mathrm{RA}=$ right atrium, $\mathrm{RAA}=$ right atrial appendage, $\mathrm{RV}=$ right ventricle, $\mathrm{RVOT}=$ right ventricular outflow tract, IVS = interventricular septum (blue dashed line), IVC = inferior vena cava, SVC $=$ superior vena cava.

References:

1. Vida VL, Padalino MA, Boccuzzo G, Tarja E, Berggren H, Carrel T, et al. Scimitar syndrome: a European Congenital Heart Surgeons Association (ECHSA) multicentric study. Circulation. 2010;122(12):1159-66.

2. Rolf S, Hindricks G, Sommer P, Richter S, Arya A, Bollmann A, et al. Electroanatomical mapping of atrial fibrillation: Review of the current techniques and advances. J Atr Fibrillation. 2014;7(4):1140-.

3. Sharma PS, Dandamudi G, Herweg B, Wilson D, Singh R, Naperkowski A, et al. Permanent Hisbundle pacing as an alternative to biventricular pacing for cardiac resynchronization therapy: A multicenter experience. Heart Rhythm. 2018;15(3):413-20.

4. Ajijola OA, Upadhyay GA, Macias C, Shivkumar K, Tung R. Permanent His-bundle pacing for cardiac resynchronization therapy: Initial feasibility study in lieu of left ventricular lead. Heart Rhythm. 2017;14(9):1353-61.

5. Paparella G, Chierchia GB, Sarkozy A, Francesconi A, de Asmundis C, Capulzini L, et al. Persistent left superior vena cava in patients treated with His-bundle pacing: trouble or help? Europace. 2008;10(9):1102-4.

6. Molina-Lerma M, Jimenez-Jaimez J, Macias-Ruiz R, Sanchez-Millan P, Tercedor L, Alvarez M. Hisbundle pacing in a patient with dextrocardia, severe systolic dysfunction, and complete atrioventricular block. HeartRhythm Case Rep. 2019;5(3):148-51.

7. Segan L, Perrin M, Thomas J, Ridley D. His bundle pacing in Ebstein's anomaly. EP Europace. 2019;21(12):1856-.

8. Kean AC, Kay WA, Patel JK, Miller JM, Dandamudi G. Permanent nonselective His bundle pacing in an adult with L-transposition of the great arteries and complete AV block. Pacing Clin Electrophysiol. 2017;40(11):1313-7.

9. Moore JP, Gallotti R, Shannon KM, Pilcher T, Vinocur JM, Cano O, et al. Permanent conduction system pacing for congenitally corrected transposition of the great arteries: A Pediatric and Congenital Electrophysiology Society (PACES)/International Society for Adult Congenital Heart Disease (ISACHD) Collaborative Study. Heart Rhythm. 2020. 